\title{
A COVERING PROPERTY WHICH IMPLIES ISOCOMPACTNESS. I
}

\author{
J. M. WORRELL, JR. AND H. H. WICKE
}

\begin{abstract}
We define a covering property for a topological space which does not explicitly mention countability or finitude of collections although it generalizes weak $\delta \theta$-refinability. We prove a general theorem that implies that countably compact spaces having the covering property are compact.
\end{abstract}

1. Introduction. The main purpose of this paper is to consider a covering property which generalizes a number of those in the literature including weak $\delta \theta$-refinability. Thus it generalizes such properties as paracompactness and metacompactness. Unlike the familiar properties just mentioned, the covering property introduced here does not explicitly mention the finitude or countability of collections at a point or the possibility of expressing a cover as a countable union of certain collections. We regard this paper as a natural sequel to the paper $\left[\mathbf{W W}_{2}\right]$, which provides further background on covering properties. The following corollary of the results obtained here generalizes Theorem 2.3 of the paper $\left[\mathbf{W W}_{2}\right]$ which established the isocompactness of weak $\delta \theta$-refinability (see also [WoW], where an equivalent proposition was stated). The statement of 1.1 illustrates the point made above about mention of finitude or countability.

1.1. TheOREM. Suppose $X$ is a countably compact space such that for every open cover $\mathcal{H}$ of $X$ of uncountable regular cardinality there is a collection 2 of collections of open subsets of $X$ such that (1) $\cup \mathcal{2}$ refines $\mathcal{H},(2)|\mathcal{2}|<|\mathcal{H}|$, and (3) for each $p \in X$ there is a $\mathscr{V} \in \mathcal{Q}$ such that $0<|\{V \in \mathcal{V}: p \in V\}|<|\mathcal{H}|$. Then $X$ is compact.

From this result the isocompactness of weakly $\delta \theta$-refinable spaces (and, hence, of a number of other kinds of spaces [ $\left.\mathbf{W W}_{2}\right]$ ) follows directly.

Our approach here is to prove a main theorem concerning closed ultrafilters in spaces having a covering property related to that expressed in 1.1 and satisfying a cardinality restriction on discrete subspaces. The main theorem implies 1.1 and leads to analogues and generalizations of other results including some found in [B], $[\mathbf{H V}]$, and $\left[\mathbf{W W}_{2}\right]$.

2. Definitions and preliminaries. We establish some convenient terminology here. For definitions of covering properties not given here we refer to [ $\left.\mathbf{W}_{\mathbf{2}}\right]$.

Presented to the Society, January 25, 1979; received by the editors January 11, 1979 and, in revised form, April 21, 1979.

AMS (MOS) subject classifications (1970). Primary 54D20; Secondary 54D30.

Key words and phrases. Countably compact, compact, closed-complete, weak $\boldsymbol{\delta} \theta$-refinability, closed ultrafilters, isocompactness, (weakly) $[\alpha, \beta\}$-refinable, (weakly) $[\alpha, \infty)^{r}$-refinable.

(c) 1980 American Mathematical Society 0002-9939/80/0000-0286/\$02.00 
2.1. Definition. If $X$ is a space, $\mu$ is a cardinal number, and $\mathcal{X}$ is an open cover of $X$, a weak $[\mu]$-refinement of $\mathcal{H}$ is an open refinement of $\mathcal{H}$ (which covers $X$ ) which can be expressed as $\cup \mathcal{Q}$ where $\mathcal{Q}$ is a collection of collections of open sets which refine $\mathcal{H}$ such that: (1) $|\mathcal{2}|<\mu$, and (2) for all $p \in X$ there exists $\mathscr{V} \in \mathcal{Q}$ such that $0<|\{V \in \mathcal{V}: p \in V\}|<\mu$. A $[\mu]$-refinement is a weak $[\mu]$-refinement as above such that each member of 2 is an open cover of $X$.

The following concept of Hodel and Vaughan [HV] is clearly related to the main concept of our paper as can be seen from Definition 2.3 and the statement of Theorem 3.1.

2.2. Definition [HV]. Let $\alpha$ and $\beta$ be cardinal numbers. A space $X$ is called $[\alpha, \beta\}$-refinable if and only if for every regular cardinal $\mu$ such that $\alpha<\mu<\beta$, if $\mathcal{U}$ is an open cover of $X$ such that $|\mathscr{U}|=\mu$, then there is a $[\mu]$-refinement of $\mathscr{U}$. A space is $[\alpha, \infty)^{r}$-refinable if and only if it is $[\alpha, \beta]^{r}$-refinable for all $\beta>\alpha$.

A natural extension of the above terminology follows.

2.3. Definition. A space $X$ is weakly $[\alpha, \beta]^{r}$-refinable (respectively, weakly $[\alpha, \infty)^{r}$-refinable) if in Definition 2.2 weak $[\mu]$-refinement is used in place of $[\mu]$-refinement.

Clearly if a space is weakly $\delta \theta$-refinable, then it is weakly $\left[\boldsymbol{\kappa}_{1}, \infty\right)^{r}$-refinable. Also if every open cover of a weakly $\left[\kappa_{1}, \infty\right)^{r}$-refinable space has a subcover of cardinality $\aleph_{1}$, then the space is weakly $\delta \theta$-refinable.

3. The main theorem. Here we prove a theorem from which 1.1 and other results follow. The method of proof has some elements in common with methods used in $\left[\mathbf{W W}_{2}\right]$ and $[\mathbf{B}]$. For terminology (not given here) concerning closed ultrafilters see [B]. A closed ultrafilter $\mathscr{F}$ is said to have the $\kappa$-intersection property if every subcollection of $\mathscr{F}$ of cardinality $\leqslant \kappa$ has nonempty intersection. A closed ultrafilter $\mathscr{F}$ is called free provided $\cap \mathscr{F}=\varnothing$. As in [E], if $X$ is a space, $e(X)$ denotes the smallest cardinal $m \geqslant \aleph_{0}$ such that every closed and discrete subspace has cardinality $<m$. By a wellordered increasing (decreasing) collection of sets of cardinal $\kappa$ is meant a collection $\left\{B_{\alpha}: \alpha<\kappa\right\}$ that can be indexed by a cardinal $\kappa$ in such a way that $\alpha<\gamma<\kappa$ implies $B_{\alpha} \subseteq B_{\gamma}\left(B_{\alpha} \supseteq B_{\gamma}\right)$.

3.1. TheOREM. Suppose that $X$ is a space and $\kappa$ is an infinite cardinal such that every subset of $X$ of cardinality $>\kappa$ has a 2-limit point and every wellordered increasing open cover $\mathcal{H}$ of $X$ of regular cardinality $>\kappa$ has a weak $[|\mathcal{H}|]-$ refinement. Then no free closed ultrafilter on $X$ has the $\kappa$-intersection property.

Proof. Suppose $\mathcal{F}$ is a free closed ultrafilter on $X$ having the $\kappa$-intersection property. Let $\gamma$ be the smallest cardinal such that there exists $\mathscr{F}^{\prime} \subseteq \mathscr{F}$ with $\left|\mathscr{F}^{\prime}\right|=\gamma$ and $\cap \mathscr{F}^{\prime}=\varnothing$. Then $\kappa<\gamma$. Let $\mathscr{F}^{\prime}=\left\{F_{\alpha}: \alpha<\gamma\right\}$ where $\cap \mathscr{F}^{\prime}=\varnothing$, and define $B_{\alpha}=\cap\left\{F_{\delta}: \delta<\alpha\right\}$ for all $\alpha<\gamma$. Then $B_{\alpha} \in \mathscr{F}$ for all $\alpha<\gamma$ and $\alpha<\delta<\gamma$ implies $B_{\alpha} \supseteq B_{\delta}$ and $\cap\left\{B_{\alpha}: \alpha<\gamma\right\}=\varnothing$. Suppose $f$ is an increasing function on $\operatorname{cf}(\gamma)$ such that $\gamma=\sup \{f(\alpha): \alpha<\operatorname{cf}(\gamma)\}$. Then $\cap\left\{B_{f(\alpha)}: \alpha<\operatorname{cf}(\gamma)\right\}=\varnothing$. Hence $\operatorname{cf}(\gamma)=\gamma$, so $\gamma$ is regular. The collection $\mathcal{H}=\left\{X \backslash B_{\alpha}: \alpha<\gamma\right\}$ is a wellordered increasing open cover of $X$. Let $\mathcal{Q}$ be a collection such that $\cup \mathcal{L}$ is a weak $[|\mathcal{H}|]$-refinement of $\mathcal{H}$. Let $\mathcal{Q}=\left\{\mathfrak{V}_{\beta}: \beta<\delta\right\}$ where $\delta<\gamma$. For each $\beta<\delta$ let 
$C_{\beta}=\left\{x \in X: 0<\left|\left\{V \in \mathfrak{V}_{\beta}: x \in V\right\}\right|<\gamma\right\}$. There exists some $\beta<\delta$ such that $C_{\beta}$ intersects each element of $\mathscr{F}$. If not, then for each $\alpha<\delta$, there is $M_{\alpha} \in \mathscr{F}$ such that $C_{\alpha} \cap M_{\alpha}=\varnothing$. Since $\delta<\gamma, \cap\left\{M_{\alpha}: \alpha<\delta\right\} \in \mathcal{F}$. Since $C_{\beta} \cap \cap\left\{M_{\alpha}: \alpha<\right.$ $\delta\}=\varnothing$ for all $\beta<\delta$, it follows that $\cap\left\{M_{\alpha}: \alpha<\delta\right\}=\varnothing$, a contradiction. Let $C_{\eta}$ intersect each element of $\mathscr{F}$. For some $M \in \mathscr{F}, M \subseteq \cup \mathcal{V}_{\eta}$ (otherwise, $X \backslash \cup \mathcal{V}_{\eta}$ $\in \mathscr{F})$. By [M, Theorem 18, p. 8], there exists a set $D \subseteq M \cap C_{\eta}$ such that $M \cap C_{\eta} \subseteq \operatorname{st}\left(D, \mathcal{V}_{\eta}\right)$ and no member of $\Upsilon_{\eta}$ contains two points of $D$. Hence $D$ has no 2-limit points and the hypothesis implies that $|D|<\kappa$. Since $D \subseteq C_{\eta}$ and $\gamma$ is regular, some subcollection $\mathscr{U} \subseteq \mathcal{V}_{\eta}$ covers $M \cap C_{\eta}$ and $|\mathcal{Q}|<\gamma$. For each $U \in \mathcal{Q}$ let $H_{U} \in \mathcal{H}$ be such that $H_{U} \supseteq U$. Then $\cap\left\{X \backslash H_{U}: U \in \mathcal{U}\right\} \cap M \cap$ $C_{\eta}=\varnothing$. Since $\cap\left\{X \backslash H_{U}: U \in \mathcal{Q}\right\} \cap M \in \mathcal{F}$, this involves a contradiction.

We note that in the proof no use is made of the property that the intersection of two open sets is open (or, equivalently, the theorem can be established using only Axiom 0 of $[\mathbf{M}])$.

3.2. TheOREM. Suppose that $X$ is a $T_{1}$-space such that $e(X)<\kappa$ and every wellordered increasing open cover $\mathcal{H}$ of $X$ of regular cardinality $>\kappa$ has a weak $[|\mathcal{H}|]$-refinement. Then no free closed ultrafilter on $X$ has the $\kappa$-intersection property.

Proof. The hypothesis guarantees that $D$ in the proof of 3.1 has cardinality $<\kappa$.

The following corollary is a restatement of 1.1.

3.3. Corollary. Suppose $X$ is countably compact and weakly $\left[\aleph_{1}, \infty\right)^{r}$-refinable. Then $X$ is compact.

Proof. Since $X$ is countably compact, countably infinite subsets have $\omega$-limit points and all closed filters on $X$ have the countable intersection property. By 3.1, $X$ has no free closed ultrafilters.

3.4. Corollary [HV, Corollary 4.6]. Let $X$ be countably compact. If $X$ is $\left[\aleph_{1}, \infty\right)^{r}$-refinable, then $X$ is compact. In particular, if $X$ is either meta-Lindelöf or $\theta$-refinable, then $X$ is compact.

3.5. Corollary [ $\mathbf{W W}_{2}$, Theorem 2.3]. Every closed and countably compact subset of a weakly $\delta \theta$-refinable space is compact.

3.6. Corollary. Suppose $X$ is $\aleph_{1}$-compact and $T_{1}$ and weakly $\left[\aleph_{1}, \infty\right)^{r}$-refinable. Then $X$ is closed-complete.

Proof. The $\aleph_{1}$-compactness of $X$ implies that $e(X)<\aleph_{0}$. Closed-complete means that closed ultrafilters with the countable intersection property are not free. Thus the statement follows from 3.1.

3.7. Corollary [B, Corollary 3.3]. If $X$ is a weakly $\delta \theta$-refinable $\boldsymbol{\aleph}_{1}$-compact, $T_{1}$-space, then $X$ is closed-complete.

In the following theorem, the concept of $\theta$-diagonal is the concept called Condition II in [FL]. It is implied by each of the concepts of primitive base, 
primitive diagonal and $G_{\delta}$-diagonal. Thus the theorem generalizes several parts of Theorem 2.10 of $\left[\mathbf{W W}_{\mathbf{2}}\right]$.

3.8. TheOREM. Suppose $X$ is countably compact and $T_{2}$. Then $X$ is metrizable if and only if $X$ is weakly $\left[\aleph_{1}, \infty\right)^{r}$-refinable and has a $\theta$-diagonal.

Proof. It follows from [FL, Theorem 3.5] that compact $T_{2}$ spaces having a $\boldsymbol{\theta}$-diagonal are metrizable. Thus the result follows from Corollary 3.2.

The following theorem involves a stronger covering property which also implies weak $\delta \theta$-refinability.

3.9. THEOREM. Suppose for every uncountable open cover $\mathcal{H}$ of a space $X$ there is a collection 2 of collections of open sets such that (1) each element of 2 refines $\mathcal{H},(2)$ $|\mathcal{Q}|<|\mathcal{H}|$, and (3) for all $p \in X$, there is $\mathfrak{T} \in \mathcal{2}$ such that $0<|\{V \in \mathcal{V}: p \in V\}|$ $<|2|$. If $\kappa$ is an infinite cardinal such that every discrete subspace of $X$ has cardinality $<\kappa$, then every open cover of $X$ has a subcover of cardinality $<\kappa$ (i.e. $X$ is $\left[\kappa^{+}, \infty\right)$-compact $)$.

Proof. Suppose $\mathcal{H}$ is an open cover of $X$ of minimum cardinality that has no subcover of cardinality $\leqslant \kappa$. Then there exists a collection $\mathcal{Z}$ as described in the statement of the theorem. Let $|\mathcal{Q}|=\gamma$, and $\mathcal{L}=\left\{\mathfrak{V}_{\beta}: \beta<\gamma\right\}$. Let $C_{\beta}$ be defined as in the proof of 3.1. Then by [M], for each $\beta$ there exists a discrete $D_{\beta} \subseteq C_{\beta}$ such that $C_{\beta} \subseteq \operatorname{st}\left(D_{\beta}, \mathscr{V}_{\beta}\right)$. Since each $\left|D_{\beta}\right| \leqslant \kappa, X$ is covered by a subcollection $W$ of $\cup \mathcal{Q}$ of cardinality $\leqslant \kappa \cdot|\mathcal{L}|<|\mathcal{H}|$. Since $\cup \mathcal{Q}$ is a refinement of $\mathcal{H}$, $\mathscr{W}$ has no subcover of cardinality $\leqslant \kappa$. This contradicts the minimum cardinality of $\mathcal{H}$.

3.10. Corollary [B], [WW $]$. If $X$ is a hereditarily $\aleph_{1}$-compact $T_{1}$-space which is weakly $\delta \theta$-refinable, then $X$ is Lindelöf.

\section{REFERENCES}

[B] R. L. Blair, Closed-completeness in spaces with weak covering properties, Set-Theoretic Topology, Academic Press, New York, 1977, pp. 17-45.

[E] R. Engelking, General topology, Polish Scientific Publishers, Warsaw, 1977.

[FL] P. Fletcher and W. F. Lindgren, O-spaces, General Topology and Appl. 9 (1978), 139-153.

[HV] R. E. Hodel and J. E. Vaughan, A note on [a,b]-compactness, General Topology and Appl. 4 (1974), 179-189.

[M] R. L. Moore, Foundations of point set theory, rev. ed., Amer. Math. Soc. Colloq. Publ., vol. 13, Amer. Math. Soc., Providence, R. I., 1962.

[WW ] H. H. Wicke and J. M. Worrell, Jr., The hereditary Lindelöf property, primitive structures, and separable metrizability, Notices Amer. Math. Soc. 22 (1975), A-425.

$\left[\mathrm{WW}_{2}\right]$ H. H. Wicke and J. M. Worrell, Jr., Point-countability and compactness, Proc. Amer. Math. Soc. 55 (1976), 427-431.

[WoW] J. M. Worrell, Jr. and H. H. Wicke, Characterizations of developable topological spaces, Canad. J. Math. 17 (1965), 820-830.

Institute for Medicine ANd Mathematics, OHo University, Athens, OHo 45701

Department of Mathematics, Ohio University, Athens, Oho 45701 